\title{
Analysis of some sepsis markers: C-reactive protein, Procalcitonin, Osteopontin and suPAR
}

Ilaria Crespi', Vesselina Kroumova', Elena Grossini', Maria Giuseppina Pellò', Marzia Tozzini', Giacomo Fortina', Silvia Valsecchi ${ }^{2}$, Rosanna Vaschetto ${ }^{2}$, Francesco Della Corte ${ }^{2}$, Annalisa Chiocchietti ${ }^{3}$

I Laboratorio di Microbiologia e Virologia, Azienda Ospedaliero Universitaria Maggiore della Carità di Novara

2 Dipartimento di medicina clinica e sperimentale e dipartimento di anestesia e rianimazione, Università del Piemonte Orientale "A. Avogadro" 3 Dipartimento di scienze mediche e dipartimento di immunologia, Università del Piemonte Orientale "A. Avogadro"

Key words: Sepsis, C-rective Protein, Procalcitonin, Osteopontin, suPAR

Analisi di alcuni marcatori di sepsi: proteina C reattiva, Procalcitonina, Osteopontina e suPAR

\section{SUMMARY}

Sepsis appears to be due to an uncontrolled pro and anti-inflammatory response leading to organ dysfunction until shock and death.Accuracy of diagnosis and appropriate treatment affect the outcome. Aim of the study was to investigate if the levels of Osteopontin (OPN) and Soluble Urokinase-type Plasminoge Activator Receptor (suPAR) might be early markers of sepsis and if these markers play a role in predicting the progression to septic shock.

Methods:The levels of OPN, suPAR, C-reactive protein (CRP) and procalcitonin (PCT) were measured in patients at higher risk of infection. The samples were collected from the day of admission for the following fifteenth day. During recovery Sistemic Inflammatory Response Syndrome (SIRS) criteria, blood chemistry data, blood gas- analysis, lactate and diuresis were collected. Microbiological culture were performed according to the clinical condition.

Results: 29 patients were enrolled in the study, including 14 subjects with positive blood cultures. The values of OPN, PCT, suPAR and CRP were significantly increased in patients with positive blood cultures than those with negative blood cultures. Analysing changes of the levels of OPN, suPAR, PCT and CRP in patients with septic shock, the values of OPN increased in advance to the clinical diagnosis of septic shock, while the values of suPAR and PCT slight delay compared to those OPN.The levels of CRP were independent from the onset of septic shock state.

Conclusions: These preliminary data suggest that Osteopontin and suPAR can be used as early markers of sepsis and might be useful in monitoring of septic outcome to predict a possible evolution to septic shock.

\section{INTRODUZIONE}

Per SIRS (sindrome da risposta infiammatoria sistemica) si intende una risposta clinica a seguito di un insulto aspecifico, comprendente 2 o più dei seguenti segni clinici: temperatura $>38^{\circ} \mathrm{C} \mathrm{o}<36^{\circ} \mathrm{C}$, frequenza cardiaca $>90 \mathrm{battiti} / \mathrm{min}$, frequenza respiratoria $>20 /$ min o $\mathrm{PaCO}_{2}<32 \mathrm{mmHg}$, conta dei leucociti $>12.000 / \mathrm{mm}^{3}$ o $<4.000 / \mathrm{mm}^{3}$ o neutrofili immaturi (cellule "a bande") $>10 \%$.

La SIRS diventa SEPSI se si aggiungono segni di infezione documentata; se subentrano anche 1 o più segni di disfunzione d'organo (cardiaci, renali, respiratori, ematologici, acidosi lattica, epatici, SNC) allora si definisce sepsi severa o shock septico e a seguire la complicanza clinica definita come MODS (Multiple Organ Dysfunction Syndrome) (7). La patogenesi è molto complessa ed è dovuta ad un'attivazione incontrollata della risposta pro ed anti-infiammatoria, per alterazione dei fisiologici meccanismi di regolazione con conseguenti disfunzioni organiche fino allo shock e alla morte $(1,6,12)$.

La tempestività, l'accuratezza della diagnosi e l'adeguatezza del successivo trattamento influenzano l'outcome. Oltre alla diagnostica batteriologica classica esistono dei marcatori di laboratorio e sistemi di classificazione per definirne la severità e la prognosi ma fino ad ora nessun marcatore ha raggiunto un unanime consenso nella diagnostica della sepsi $(5,6)$.

\section{Scopo dello studio}

Lo scopo dello studio è di valutare in pazienti ad alto rischio di sviluppare un'infezione, i livelli di marcatori di sepsi già in uso come la PCR (Proteina C Reattiva) e la PCT (Procalcitonina) e di stabilire inoltre se, OPN (Osteopontina) e suPAR, proteine considerate "nuovi" marcatori possano essere utilizzate per la diagnosi precoce di sepsi e per predirne l'evoluzione verso lo shock settico $(8,9,10,11,13)$.

\section{MATERIALI E METODI}

I livelli di OPN, suPAR, Proteina C Reattiva (PCR) e Procalcitonina (PCT) sono stati misurati in pazienti ad alto rischio di sviluppare infezione. I campioni ematici sono stati raccolti dal giorno del ricovero fino al quindicesimo giorno di degenza. Durante il ricovero sono stati raccolti i dati demografici e giornalmente i criteri di SIRS, esami ematochimici, emogasanalitici, lattati e diuresi. Gli esami colturali sono stati eseguiti in base alle condizioni cliniche del paziente. La presenza di batteriemia all'emocoltura è stata messa in relazione, con il Mann-Whitney test, ai livelli di OPN, suPAR, PCT e PCR. Per la quantificazione della PCR si è usato un test al lattice (Siemens Healthcare LIASON Diagnostic, Milano, Italia) con una metodica nefelometrica; per testare la PCT si è utilizzato il kit BRAHMS PCT con una metodica in chemiluminescenza (Diasorin, Saluggia, Italia); per il test suPAR si è utilizzato il kit su PARGNOSTIC (Bio Exe, Verona, Italia) con una metodica immunoenzimatica (2, $3,4)$, seguendo le indicazioni delle ditte produttrici.

\section{RISULTATI}

Lo studio è stato condotto su 29 pazienti, di cui 14 con riscontro di emocoltura positiva. I valori di OPN, PCT, suPAR e PCR sono risultati significativamente aumentati $(\mathrm{p}<0.05)$ (Figura I) nei pazienti con emocolture positive rispetto a quelli con emocolture negative (OPN: $1573 \pm 1241$ $\mathrm{ng} / \mathrm{ml}$ vs. $790.1 \pm 620.8 \mathrm{ng} / \mathrm{ml}$; PCR: $1554 \pm 962.8 \mathrm{mg} / \mathrm{dl}$ vs. $911 \pm 891.3 \mathrm{ng} / \mathrm{ml}$; suPAR: $8.0 \pm 7.0 \mathrm{ng} / \mathrm{ml}$ vs. $3.3 \pm 1.6$ $\mathrm{ng} / \mathrm{ml}$; PCT: $7.7 \pm 3.0 \mathrm{ng} / \mathrm{ml}$ vs. $0.8 \pm 2.2 \mathrm{ng} / \mathrm{ml}$ ). Dall'analisi delle variazioni dei livelli di OPN, suPAR, PCT e PCR nei pazienti in shock settico si è osservato che i valori di OPN aumentano $5 \pm 1.4$ giorni prima della diagnosi clinica dello shock settico. I valori di suPAR e PCT (Figura II, III) aumentano invece il giorno prima della comparsa dello stato di shock mentre i livelli di PCR sembrano indipendenti dalla comparsa dello stato settico.

\section{Corresponding author: Crespi llaria}

Laboratorio di Microbiologia e Virologia,Azienda Ospedaliero Universitaria Maggiore della Carità di Novara

Corso Mazzini, 18 - 28100 Novara - Tel.: 03213733595 - Fax:032I 3733588

E-mail: ilaria.crespi@libero.it 


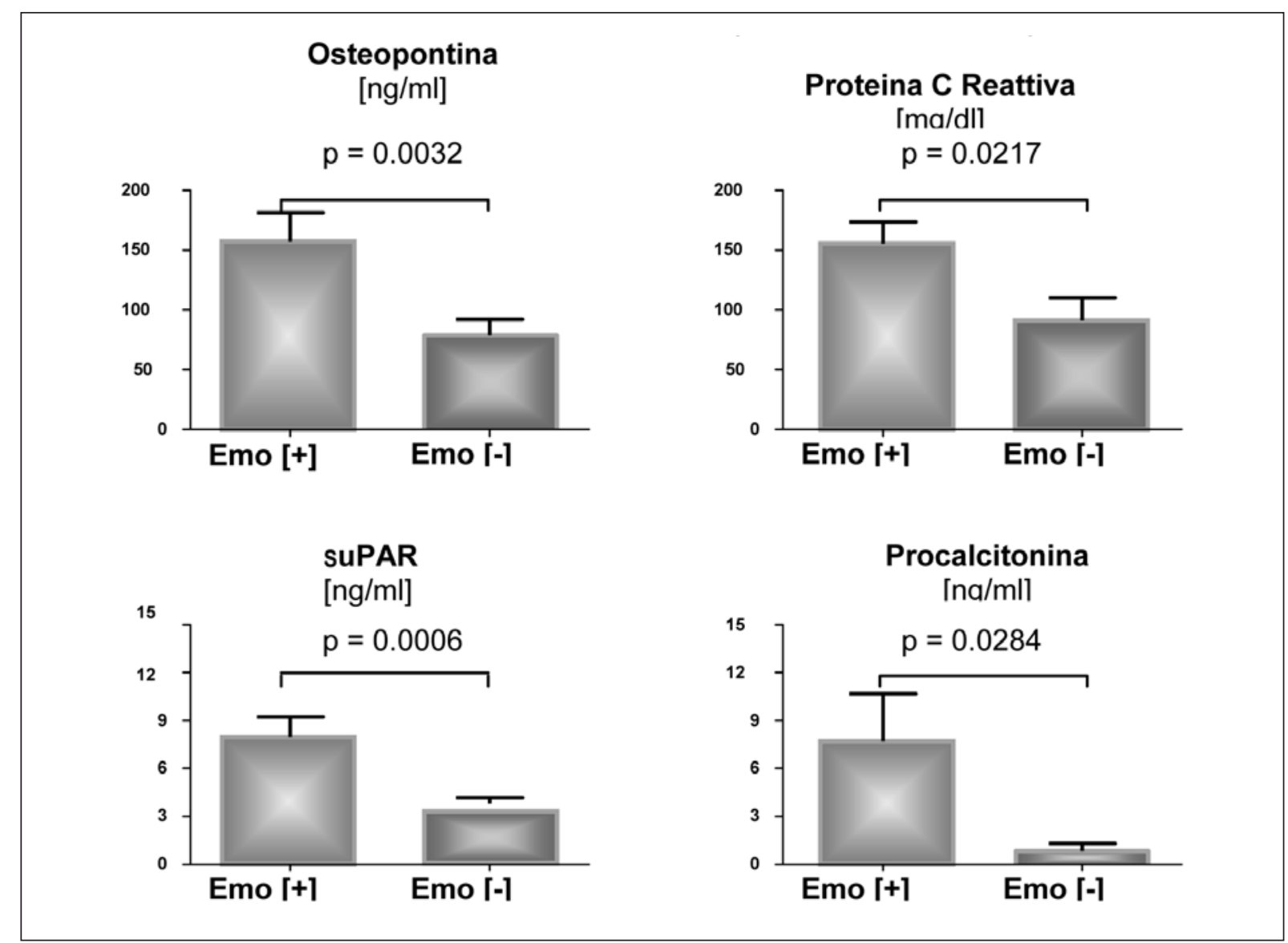

Figura I. Livelli di OPN, suPAR, PCT e PCR ed emocoltura

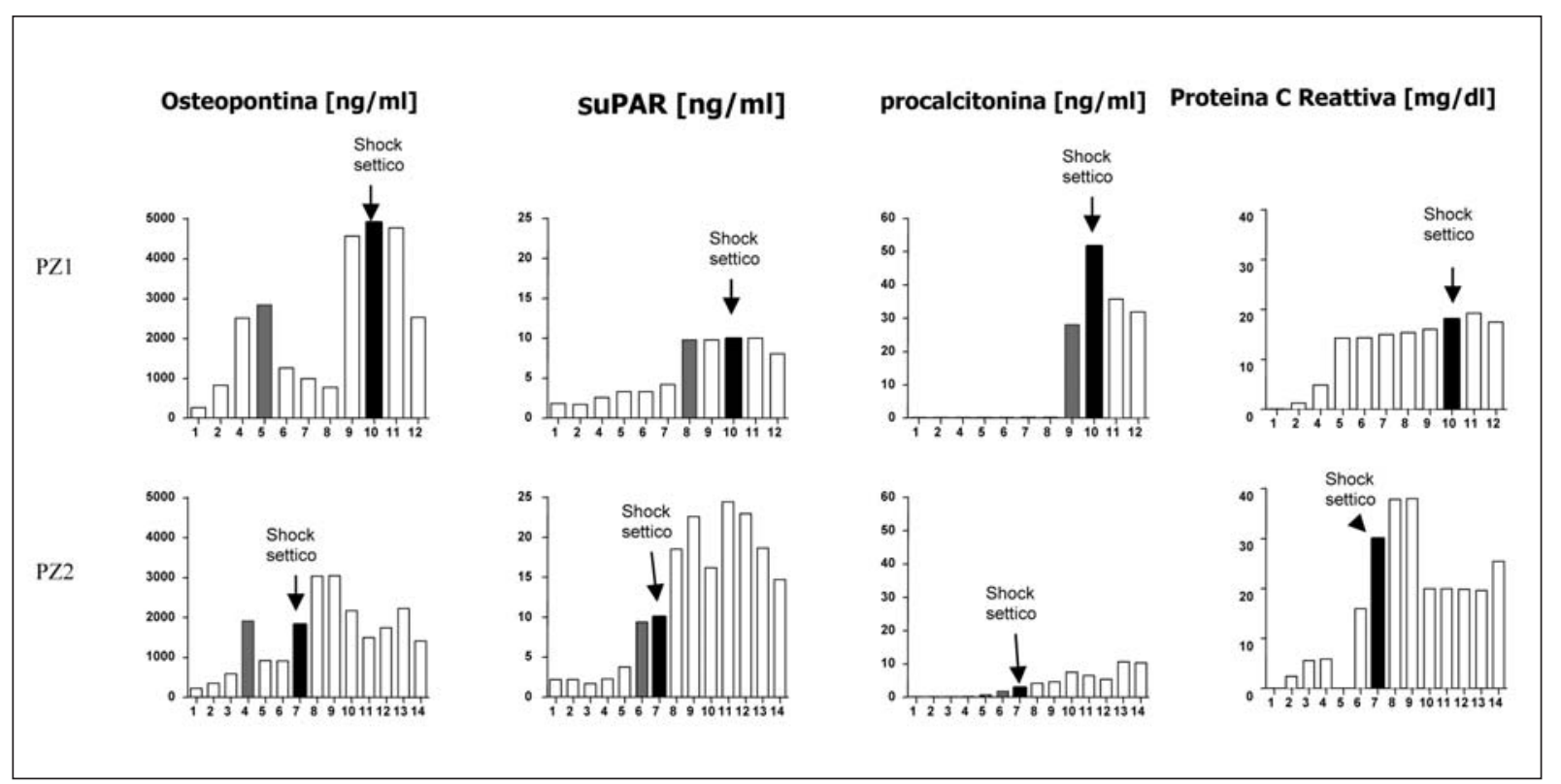

Figura II. Livelli di opn, supar, pct e pcr e shock settico

\section{CONCLUSIONI}

I nostri dati preliminari supportano un possibile ruolo dell'Osteopontina e di suPAR come marcatori di sepsi. Queste due molecole, inoltre, sembrano in grado di indicare in anticipo un'evoluzione negativa del quadro settico e, conseguentemente, potrebbero meglio monito- re tale patologia. Questi dati, seppur interessanti, necessitano di ulteriori approfondimenti che consentano di meglio conoscere le dinamiche di queste due molecole in una situazione clinica così delicata come quella della sepsi e determinare i limiti e i vantaggi di un loro utilizzo. 


\section{BIBLIOGRAFIA}

1. Adib-Conquy M, Cavaillon JM. Stress molecules in sepsis and systemic inflammatory response syndrome. FEBS Letters 2007; 581: 3732-3.

2. Florquin S, van den Berg JG, Olszyna DP, et al. Release of urokinase plasminogen activator receptor during urosepsis and endotoxemia. Kidney International, 2001; 59: 2054-61.

3. Kofoed K, Andersen O, Kronborg G, et al. Use of plasma C-reactive protein, procalcitonin, neutrophils, macrophage activator receptor, and soluble triggering receptor expressed on myeloid cells-1 in combination to diagnose infections: a prospective study. Critical Care, 2007; 11 (2): 11: R38.

4. Kofoed K, Schneider UV, Scheel T, Andersen O, Eugen-Olsen J. Development and validation of a multiplex add-on assay for sepsis biomarkers using xMAP technology. Clinical Chemestry, 2006; 52 (7); 1284-93.

5. Lam HS, Pak CNG. Diagnostic markers in neonatal sepsis. Fetal and Maternal Medicine Review, 2007; 18 (I): 53-65.

6. Meisner M. Biomarkers of sepsis: clinically useful?. Curr Opin in Critic Care, 2005; 11 (5); 473-80.

7. Mitaka C. Clinical laboratory differentiation of infectious versus non- infectious systemic inflammatory response symdrome. Clinica Chimica Acta, 2005; 351: 17-29.

8. Mondino A, Blasi F. uPA and suPAR in fibrinolysis, immunity and pathology. TRENDS in Immunology, 2004; 25 (8): 450-5.

9. Ostergaard C, Benfield T, Lundgren JD, Eugen-Olsen J. Soluble urokinase receptor is elevated in cerebrospinal fluid from patients with purulent meningitis and is associated with fatal outcome. Scand Jorn Infec Dis, 2004; 36 (1): 9-14.

10. Simon L, Gauvin F, Amre DK, Saint-Louis P, Lacroix J. Serum procalcitonin and C-reactive protein levels as markers of bacterial infection: a systematic review and meta-analysis. Clin Infect Dis 2004; 39: 206-17.

11. Tang BMP, Eslick GD, Craig JC, McLean AS. Accuracy of procalcitonin for sepsis diagnosis in critically ill patients: systematic review and ametaanalysis. Lancet Infect Dis 2007; 7: 210-7.

12. Van Amersfoort ES, van Berkel TJC, Kuiper J. Receptors, mediators and mechanism involved in bacterial sepsis and septic shock. Clinical Microbiology Reviews, 2003; 379-414.

13. Wittenhagen P, Kronborg G, Weis N, et al. The plasma level of soluble urokinase receptor is elevated in patients with Streptococcus pneumoniae bacteraemia and predict mortality. Clin microb Infect, 2004; 10; 409-15. 